\title{
Detecting circumstellar disks around gravitational microlenses
}

\author{
M. Hundertmark, F. V. Hessman, and S. Dreizler
}

Institute for Astrophysics, University of Göttingen, Friedrich-Hund-Platz 1, 37077 Göttingen, Germany

e-mail: mhunder@astro.physik.uni-goettingen.de

Received 2 December 2008 / Accepted 23 March 2009

\section{ABSTRACT}

\begin{abstract}
Aims. We investigate the chance of detecting proto-planetary or debris disks in stars that induce microlensing event (lenses), and consider the modification of the light curve shapes due to occultation and extinction by the disks, as well as the gravitational deflection caused by the additional mass.

Methods. The magnification of gravitational microlensing events is calculated using the ray shooting method. The occultation is taken into account by neglecting or weighting the images on the lens plane according to a transmission map of the corresponding disk for a point source point lens (PSPL) model. The estimated frequency of events is obtained by considering the possible inclinations and optical depths of the disk.

Results. We conclude that gravitational microlensing can be used, in principle, as a tool for detecting debris disks beyond $1 \mathrm{kpc}$, but estimate that each year around 1 debris disk is expected for lens stars of F, G, or K spectral type and about 10 debris disks might have shown signatures in existing datasets.
\end{abstract}

Key words. gravitational lensing - astrometry - circumstellar matter - methods: data analysis

\section{Introduction}

Since the discovery of the first circumstellar dust disk around Vega by the Infrared Astronomy Satellite (Aumann et al. 1984), at least 101 circumstellar disks have been resolved around premain and main sequence stars out to a distance of $1 \mathrm{kpc}^{1}$, including 17 so-called debris disks. Proto-planetary disks may be quite common during the pre-main sequence life of a star; but with a maximum life span of only $10 \mathrm{Myr}$, a galactic star formation rate of 5 stars/yr, an upper limit of 400 billion stars in the MilkyWay (McKee \& Williams 1997; Diehl et al. 2006), the chance of a random star containing a proto-planetary disk is only about $0.01 \%$. The debris disks that remain thereafter last much longer, perhaps as long as 10 Gyr (e.g. Greaves 2005), so should be much more common. Trilling et al. (2008) have shown that the occurrence of $70 \mu \mathrm{m}$ excess emission in a sample of $\mathrm{F}, \mathrm{G}$, and $\mathrm{K}$ stars - presumedly due to dust in some form of debris disk - is $16 \pm 3 \%$. In contrast, practically no main sequence M-stars show excess $70 \mu \mathrm{m}$ emission: Rhee et al. (2007) have found only one disk (AU Mic) from a sample of 900 M-stars.

While a common phenomenon, circumstellar disks around main sequence stars can be difficult to detect using the standard methods of coronography and the detection of excess infrared emission. A third method - detecting the disks in absorption against a background source - works very well for dense protoplanetary disks seen against an emission nebula ("proplyds"; McCaughrean \& O'Dell 1996), but debris disks around main sequence stars are optically thin and unlikely to be found in front of a bright background.

Gravitational lensing could provide an additional method for detecting and characterizing circumstellar disks by creating a well-defined and bright, if temporary and geometrically complicated, background source. If the projected distance between the lens star and the background source is small enough, the source

\footnotetext{
1 http://www. circumstellardisks.org/
}

star appears to separate into two geometrically extended objects - the source of the photometric magnification in so-called microlensing, where the lensed object is not resolved. The angular scale of gravitational lensing is given by the Einstein radius

$\theta_{\mathrm{E}}=\sqrt{\frac{4 G M}{c^{2}} \frac{D_{\mathrm{LS}}}{D_{\mathrm{S}} D_{\mathrm{L}}}}$

where $D_{\mathrm{LS}}$ denotes the lens-source distance, $D_{\mathrm{L}}$ the lensobserver distance, $D_{\mathrm{S}}$ the source-observer distance, and $M$ is the lens mass. For a typical galactic microlensing event observed towards the galactic bulge (Paczyński 1991) with a $0.5 M_{\odot}$ lens at $6 \mathrm{kpc}$ and a source at $8 \mathrm{kpc}$, one obtains an angular scale of approximately a mas (Wambsganss 2006), corresponding to a distance of $\sim 2.5 \mathrm{AU}$ at the distance of the lens. Thus, the typical microlensing events studied primarily to detect low-mass exoplanets around lens stars (Beaulieu et al. 2006; Bennett et al. 2008) may also contain information about much more diffuse circumstellar matter.

Bozza \& Mancini (2002a) and Bozza et al. (2002b) have studied the effects of microlensing by lenses consisting of gas clouds and interacting binaries, which may be used as an approximation for a face-on proto-planetary disk and Zheng \& Ménard (2005) have considered the effects of disks around microlensing source stars, but to our knowledge the detectibility of circumstellar disks around lens stars has not been considered in the literature. About $70 \%$ of the observed lens stars towards Baade's window are M stars and smaller, and only $\sim 25 \%$ are of spectral type F, G, or K (Dominik 2006). Given that the microlensing survey groups OGLE (Udalski et al. 1994) and MOA (Abe et al. 1997) are detecting about 1000 events per year, the fraction of events containing a debris disk should be significant: about $4.0 \%$ or currently $\sim 40 /$ year. The chance of detecting proto-planetary disks among lens stars is correspondingly lower: $0.025 \%$ or $\sim 0.25$ per year (neglecting the additional absorption likely to be found within star-forming regions). Although only 1 

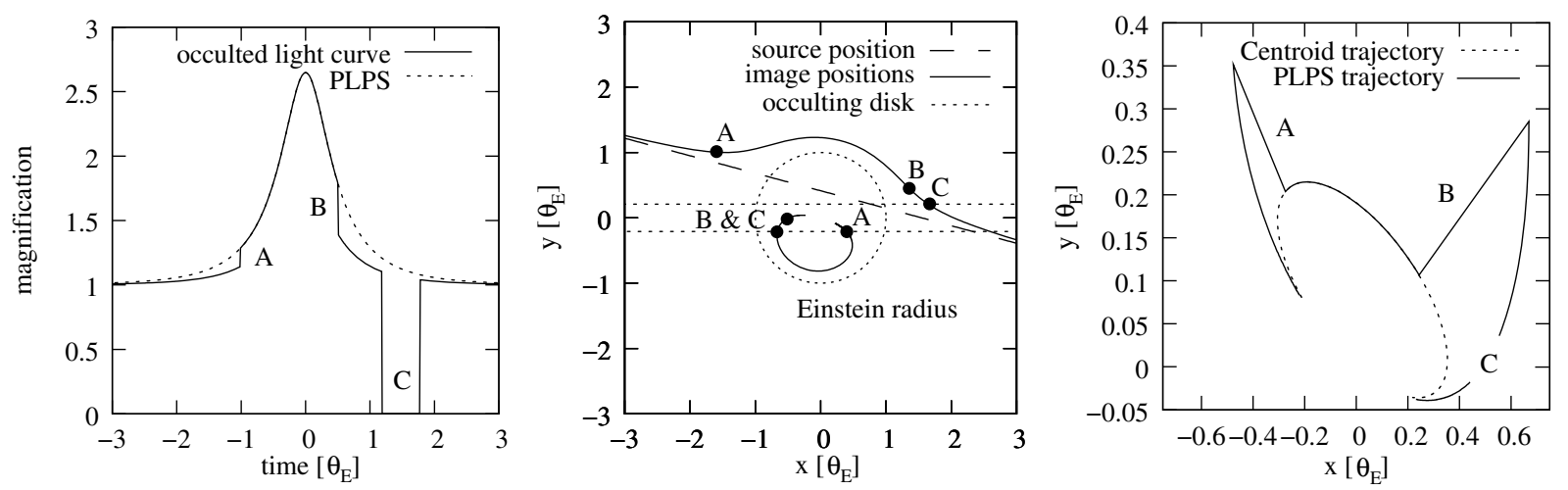

Fig. 1. Light curve of the point source point lens model occulted by an optically thick edge-on disk is plotted along with the corresponding source and image tracks and the astrometrically detectable astrometric offset from the source. Features A and B are caused by the occultation of the less magnified image while feature $\mathrm{C}$ is obtained from the occultation of both images. The impact parameter is $0.4 \theta_{\mathrm{E}}$, the inclination $89.8^{\circ}$ and the disk is rotated by $15^{\circ}$.

in 8000 lensed stars have proto-planetary disks, future surveys like the Microlensing Planet Finder (Bennett et al. 2004) or EUCLID (e.g. Beaulieu et al. 2008) have a chance of detecting a significant number of such rare events.

The effects of a circumstellar disk on microlensing light curves can be separated into two very different regimes. The disk can occult or at least extinct the light of the source star as it is bent around the lens star - a simple geometric effect given a description of the geometry and matter distribution of the disk and the geodesic paths of the photons from the source. Alternatively, the mass of the disk itself can distort the geodesic paths of the photons and hence change the apparent areas of the distorted source images and the resulting light curve independent of any occultation or extinction effects. The purely geometric disk parameters are the projected size, shape, inclination, and orientation angle $\phi$ of the major axis of the disk relative to the path of the source star and the size of the lens star's Einstein radius. The typical outer radii of proto-planetary disks are about 100$800 \mathrm{AU}$ and of debris disks about 50 to $100 \mathrm{AU}$, (e.g. Greaves 2005) corresponding to 20-320 $\theta_{\mathrm{E}}$ for a typical lens. In both cases we adapt an outer radius of $150 \mathrm{AU}$ as chosen by Brauer et al. (2008).

In the following we discuss the chance of detecting circumstellar disks by simulating a variety of potential light curves. We will start with optically thick disks, where the optical depth is greater than one (cf. Krivov 2005) representing the young protoplanetary disk phase and show that the corresponding magnification structure is scaled according to the ratio of the disk mass and its stellar host. This insight leads us to the most likely case of a microlens surrounded by an old debris disk modeled as optically thin and geometrically thick attenuating disks with optical depths $\tau \approx 0.01$.

\section{Optically thick proto-planetary disks}

Given that the major microlensing effects occur for impact parameters less than a few Einstein radii, a magnified background source will only be visible behind proto-planetary disks with high inclinations; disks with low inclinations may be too large to show any light deflection effects and will only show simpler occultations of non-magnified sources. Similarly, debris disks with high inclinations are more likely to be detected because both the projected extinction and the projected mass-density responsible for any additional lensing effect will be higher.
An optically thick disk can be modeled as an occulting disk with given inclination and orientation angles as parameters. The features of the corresponding light curves and the astrometrically observable change of the centroid (see Han \& Lee 2002) of both images are illustrated in Fig. 1, neglecting the gravitational deflection due to the disk mass. The less magnified image is occulted close to the maximum of magnification. Because the disk structure is bound to the lens, it is unlikely to occult the magnified image exclusively, unless the edge-on-disk is nearly parallel to the straight line through the two images and the lens. An asymmetric total occultation of both images as in Fig. 1 indicates that an optically thick disk structure is present.

In addition to the purely photometric effect, the occultation also produces a shift in the center-of-light position of the lensed source, albeit small. The astrometric offset due to the occultation of the less magnified images, without parallax effects (see Gould 1992), can be estimated by assuming that these images are located close to the Einstein radius

$\delta \theta \approx \frac{\mu_{-}}{\mu} \theta_{\mathrm{E}}=\frac{1}{2}\left(1-\frac{u \sqrt{u^{2}+4}}{u^{2}+2}\right)$

where $u$ denotes the lens-source separation in $\theta_{\mathrm{E}}, \mu$ the total magnification and $\mu_{ \pm}$the magnification factor of magnified and demagnified image. For a typical galactic microlensing event, the angular Einstein radius is $\approx 0.4$ mas: if the source-lens separation is below 1 Einstein radius, the pre-factor in Eq. (2) takes values between 0.12 and 0.5 and the total offset is of the order of $10 \mu$ as Fig. 1), which is detectable using the planned GAIA (Lindgren \& Perryman 1996) or SIM (Paczynski 1998; Unwin et al. 2008) satellites. For ground-based observations Rattenbury \& Mao (2006) propose using closure phase interferometry, which could provide the desired accuracy in this context.

Given the rarity of such young disks and the difficulty of seeing microlensing effects, detecting such disks photometrically and astrometrically is not impossible but unlikely.

\section{Optically thin debris disks}

\subsection{Geometrically thin disks}

Up to now, we have neglected the gravitational deflection by the mass content of the circumstellar disk, represented by the mass surface density

$\Sigma \approx 0.04 \mathrm{~g} \mathrm{~cm}^{-2}\left(\frac{q_{\mathrm{D}}}{0.001}\right)\left(\frac{M_{\star}}{0.5 M_{\odot}}\right)\left(\frac{R_{\mathrm{D}}}{150 \mathrm{AU}}\right)^{-2}\left(\frac{r}{R_{\mathrm{D}}}\right)^{-0.8}$ 


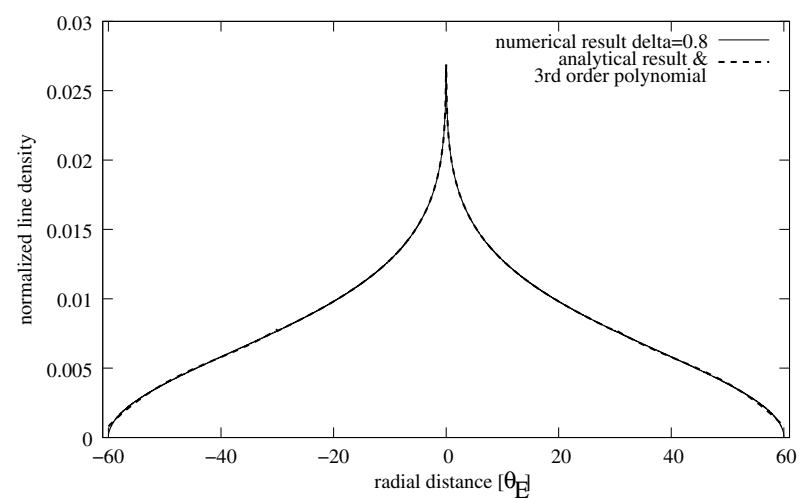

Fig. 2. The normalized line density of an edge-on oriented disk with a surface density $\Sigma \propto\left(\frac{r}{1 \mathrm{AU}}\right)^{-0.8}$, and a disk with $r_{\text {in }}=0.03 \mathrm{AU}$ and $\theta_{\mathrm{D}}=60 \theta_{\mathrm{E}}$.

(e.g. Brauer et al. 2008), where $q_{\mathrm{D}} \equiv M_{\mathrm{D}} / M_{\star}$ is the ratio of the disk mass to the stellar mass, and $R_{\mathrm{D}}$ is the effective outer radius.

Assuming $q_{\mathrm{D}}$ is small, the maximum change in deflection angle and magnification is for edge-on oriented disks, whose effective linear mass distribution can be calculated by integrating the surface density. The deflection angle of the combined point source point lens (PSPL) and edge-on disk model has no rotational symmetry, so we have to consider the vectorial deflection angle $\alpha$. An analytical expression can be derived if one integrates the normalized line density in the lens plane $\lambda\left(\theta_{1}\right)$ along the disk radius $\theta_{\mathrm{D}}$, expressed in units of the Einstein radius (cf. Schneider et al. 1992):

$\boldsymbol{\alpha}(\boldsymbol{\theta})=\frac{1}{\left(1+q_{\mathrm{D}}\right)} \cdot \frac{1}{|\boldsymbol{\theta}|}+\frac{q_{\mathrm{D}}}{q_{\mathrm{D}}+1} \int_{-\theta_{\mathrm{D}}}^{\theta_{\mathrm{D}}} \lambda\left(\theta_{1}^{\prime}\right) \frac{\left(\boldsymbol{\theta}-\boldsymbol{\theta}^{\prime}\right)}{\left|\boldsymbol{\theta}-\boldsymbol{\theta}^{\prime}\right|^{2}} \mathrm{~d} \theta_{1}^{\prime}$.

The normalized line density for a mass distribution parallel to $\theta_{1}$ is given by

$\lambda\left(\theta_{1}\right)=\int_{-\sqrt{\theta_{\mathrm{D}}^{2}-\theta_{1}^{2}}}^{\sqrt{\theta_{\mathrm{D}}^{2}-\theta_{1}^{2}}}\left(\theta_{1}^{2}+\theta_{3}^{\prime 2}\right)^{-0.4} \mathrm{~d} \theta_{3}^{\prime}$

where $\theta_{1,2}$ define the lens plane and the integral over $\theta_{3}$ projects the surface density distribution onto the line. The resulting expression contains the hypergeometric function ${ }_{2} F_{1}$ (Abramowitz \& Stegun 1972):

$$
\begin{aligned}
\lambda\left(\theta_{1}\right)= & \left|\theta_{1}\right|^{1 / 5}\left[0 . 4 5 \left(22.18\left(\theta_{\mathrm{D}}^{2}-\theta_{1}^{2}\right)^{1 / 10} \theta_{1}^{-2 / 10} .\right.\right. \\
& \left.\left.{ }_{2} F_{1}\left(-\frac{1}{10} ; \frac{4}{10} ; \frac{9}{10} ; \frac{\theta_{1}^{2}}{\theta_{\mathrm{D}}^{2}-\theta_{1}^{2}}\right)-18.94\right)\right],
\end{aligned}
$$

which can be efficiently evaluated when separated far enough from the singularities at $\pm \theta_{\mathrm{D}}$. When a third-order polynomial fit is used outside a $40 \theta_{\mathrm{E}}$ radius, as shown in Fig. 2, the total deflection angle (Eq. (4)) can be numerically integrated.

Even though the lens equation with the additional term for the deflection angle cannot be solved analytically, the disk can be embedded in a ray shooting framework (Kayser et al. 1986, Wambsganss 1997, 1999). For $q_{\mathrm{D}} \sim 0.2$, the calculated magnification offset within $1 \theta_{\mathrm{E}}$ from the disk is $\sim 1$; hence potentially observable. Close to the linear mass distribution representing the disk, one sees an additional rectangular magnification caused by the attraction of rays (Fig. 3). For small source-lens separations $u$ (here $u<0.05 \theta_{\mathrm{E}}$ ), the singular peak splits in two in the central region. For $q_{\mathrm{D}} \sim 0.20$, the affected region of the extended box is
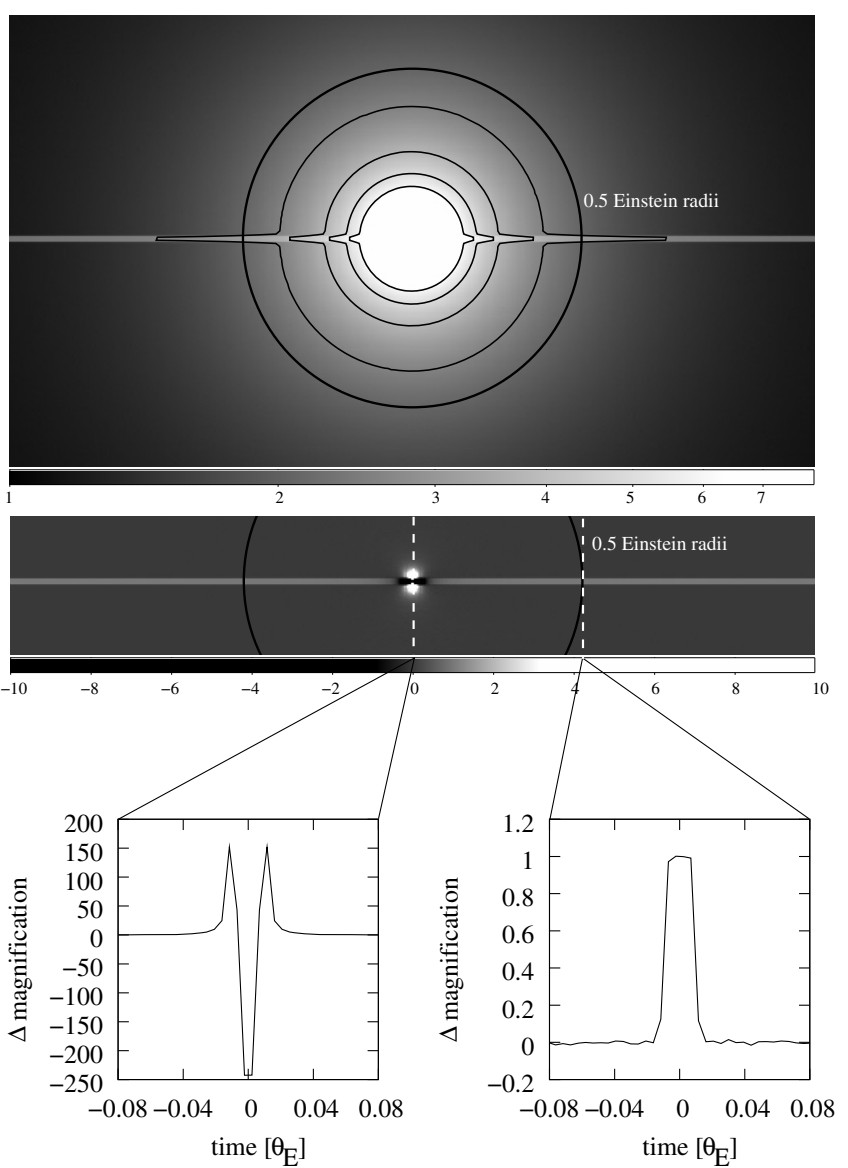

Fig. 3. Top: magnification map for a linear mass distribution created by ray shooting (Eq. (4)) for the combination of a point lens and a disk mass of $20 \%$ of the stellar host along with contour lines of magnifications at $\mu=2.5,3.7,4.8$, and 6 . Profiles extracted from the magnification map located in the source plane correspond to light curves. Middle: change relative to a point source point lens (PSPL) model. Bottom: vertical cuts corresponding to deviations in the light curves for two perpendicular trajectories of the lens and source for $0,0.5 . \theta_{\mathrm{E}}$.

$0.02 \theta_{\mathrm{E}}$ and the central distortion has a relative deviation greater than $1 \%$ for $0.2 \theta_{\mathrm{E}}$, where the Einstein radius is calculated for the total mass of the system.

According to Eq. (4), the width of the distortion is proportional to $\lambda\left(\theta_{1}\right)$ and thus to the mass ratio $q_{\mathrm{D}}$ for $q_{\mathrm{D}} \ll 1$. The rectangular distortion is redistributed in case of an inclined disk due to the conservation of rays: for $i=89^{\circ}$ the conserved integral of the rectangular distortion reduces the peak deviation to $2 \%$ and a face-on rotational symmetric disk has no effect at all. If the disk has no central gap, a deviation containing a split singularity is observable as long as the finite size of the source star does not smooth it out. Considering a bright giant source star with $10 R_{\odot}$ and thus $0.014 \theta_{\mathrm{E}}$ at $8 \mathrm{kpc}$, this limit is reached at a mass ratio of 0.014 and, in the case of inclined disks, even earlier.

Calculating the extinction in a geometrically and optically thin debris disk requires an estimation of the vertical optical depth $\tau_{\lambda}(r)=\kappa_{\lambda} \Sigma(r)$. The mean $R$-band optical depth of AU Mic is in the range $10^{-3}$ to $10^{-4}$ (Kalas 2004). Using Eq. (3) and an area-weighting of $\tau$, one obtains an estimate of the extinction coefficient (assumed to be constant)

$\kappa_{R} \approx 0.016 \mathrm{~cm}^{2} \mathrm{~g}^{-1}\left(\frac{\overline{\tau_{R}}}{10^{-3}}\right)\left(\frac{q_{\mathrm{D}}}{0.001}\right)^{-1}\left(\frac{M_{\star}}{0.5 M_{\odot}}\right)^{-1}$ 


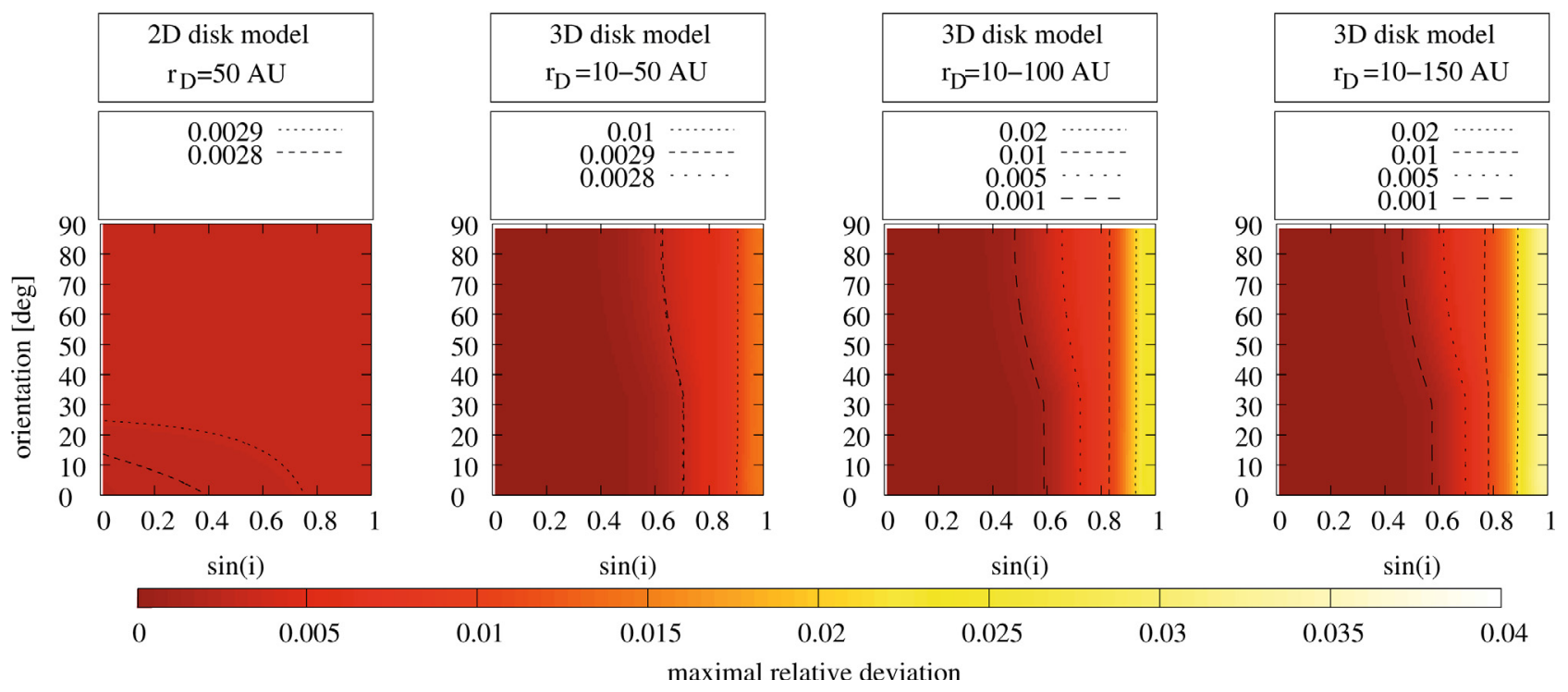

Fig. 4. Contour lines of maximal relative deviation from PSPL and a grid of attenuated light curves with $u_{0}=0.1 \theta_{\mathrm{E}}$ are plotted for a surface density $\Sigma \propto r^{-0.8}$, different outer disk radii $r_{\mathrm{D}}=50,100,150 \mathrm{AU}$, an inner disk radius of $10 \mathrm{AU}$, and a constant mean optical depth of $\bar{\tau}=5 \times 10^{-4}$.

and a final $R$-band optical depth distribution

$\tau_{R}(r)=6 \times 10^{-4}\left(\frac{\overline{\tau_{R}}}{10^{-3}}\right)\left(\frac{R_{\mathrm{D}}}{150 \mathrm{AU}}\right)^{-2}\left(\frac{r}{R_{\mathrm{D}}}\right)^{-0.8}$.

For a typical inner disk radius of $0.03 \mathrm{AU}, \tau_{R}$ is smaller than unity even in the disk center. A thin disk with such low optical depths produces relative deviations of the light curve on the order of $0.1 \%$. Current ground-based microlensing observations require an order of magnitude higher deviation and so could detect an average optical depth $>5 \times 10^{-3}$; according to Augereau $\&$ Beust (2006). This would be the maximal optical depth in the visible for the AU Mic debris disk, transversed in the vertical direction, but for an edge-on orientation, optical depths of $4 \times 10^{-2}$ could be reached. Consequently, edge-on configurations are much more likely to be detected from microlensing, too.

\subsection{Geometrically thick disks}

The attenuating effect of a circumstellar disk depends especially on the path length of the light ray through the disk and thus on its shape. For an edge-on configuration and a $0.5 M_{\odot}$ star of $0.6 R_{\odot}$, the deflection causes a vertical shift of $10^{-3} \mathrm{AU}$ at $150 \mathrm{AU}$ in the vertical direction and can be neglected. In the following, a geometrically thick disk with a Gaussian vertical dust distribution and a scale height $H(r)=0.47\left(\frac{r}{50 \mathrm{AU}}\right)+0.34 \mathrm{AU}$ is used (for AU Mic cf. Metchev et al. 2005; Krist et al. 2005). The density $\rho$ of the disk is given by

$\rho(r, z)=\frac{\Sigma(r)}{H(r) \sqrt{2 \pi}} \mathrm{e}^{-z^{2} / 2 H(r)}$.

The normalization constant is determined by integrating $\rho$ parallel to the disk normal

$\tau_{\mathrm{R}}=5.07 \times 10^{-6} \int_{0}^{s} \rho(r, z) \mathrm{d} s$.

In PSPL models the light path is usually parameterized using two straight lines according to the thin lens approximation. To first order, the true light path is a hyperbola: according to Gönner (1996, p. 206), the maximal deviation of the distance of closest approach $R$ from the hyperbola is

$\Delta R=\frac{2 G M R}{R c^{2}+2 G M}$

where $M$ is the mass of the deflecting star. The maximal deviation from the thin lens approximation for grazing incidence is $10^{-5} \mathrm{AU}$ at $0.6 R_{\odot}$ and a deflector mass of $0.5 \mathrm{M}_{\odot}$.

The sensitivity of different models for $\tau \sim 5 \times 10^{-4}$ is shown in Fig. 4. The source track for each pair of $(i, \phi)$ was limited to a range from -1 to $1 \theta_{\mathrm{E}}$ and the impact parameter $u_{0}=0.1 \theta_{\mathrm{E}}$ has been kept fixed. It does not change the maximal variation of the transmissivity, only the maximal distance from the center. While the two-dimensional model (projected surface density) with $\Sigma \propto r^{-0.8}$ shows a maximal deviation below $1 \%$, the more realistic three-dimensional model with $\Sigma \propto r^{-0.8}$ shows an increased deviation at high inclinations, because the transversed matter is taken into account. The smaller the outer radius of the disk, the poorer the detectability. From the calculated sensitivity pattern the fraction of the parameter space exceeding $1 \%$, i.e. $10 \mathrm{mmag}$, can be calculated: $17 \%$ for $r_{\mathrm{D}}=50 \mathrm{AU}, 34 \%$ for $r_{\mathrm{D}}=100 \mathrm{AU}$, and $50 \%$ for $r_{\mathrm{D}}=150 \mathrm{AU}$. These results are valid for a system without any inner clearing, but Wyatt et al. (2007) have concluded from observations that $2 \pm 2 \%$ show hot dust close to the star. Assuming an inner radius of $10 \mathrm{AU}$ changes the detectability to $10 \%$ for $r_{\mathrm{D}}=50 \mathrm{AU}$, to $18 \%$ for $r_{\mathrm{D}}=100 \mathrm{AU}$, and to $23 \%$ for $r_{\mathrm{D}}=150 \mathrm{AU}$. Changing the scale height redistributes the sensitivity to smaller inclinations.

In Fig. 5, five different sensitivity configurations are calculated for $r_{\mathrm{D}}=50 \mathrm{AU}$ and an inner gap with $10 \mathrm{AU}$ radius. Simulations for $100 \mathrm{AU}$ and $150 \mathrm{AU}$ show similar results: all of them show a systematic decrease in the residuals close to the maximal magnification, especially at high inclination. The source and image tracks plotted over the map of transmissivity explain this behavior for a surface density modeled as an inverse power law. The image with positive parity reaches its maximal separation from the lens when the source-lens distance is minimal, therefore the optical depth $\propto \Sigma$ is also minimal. This 

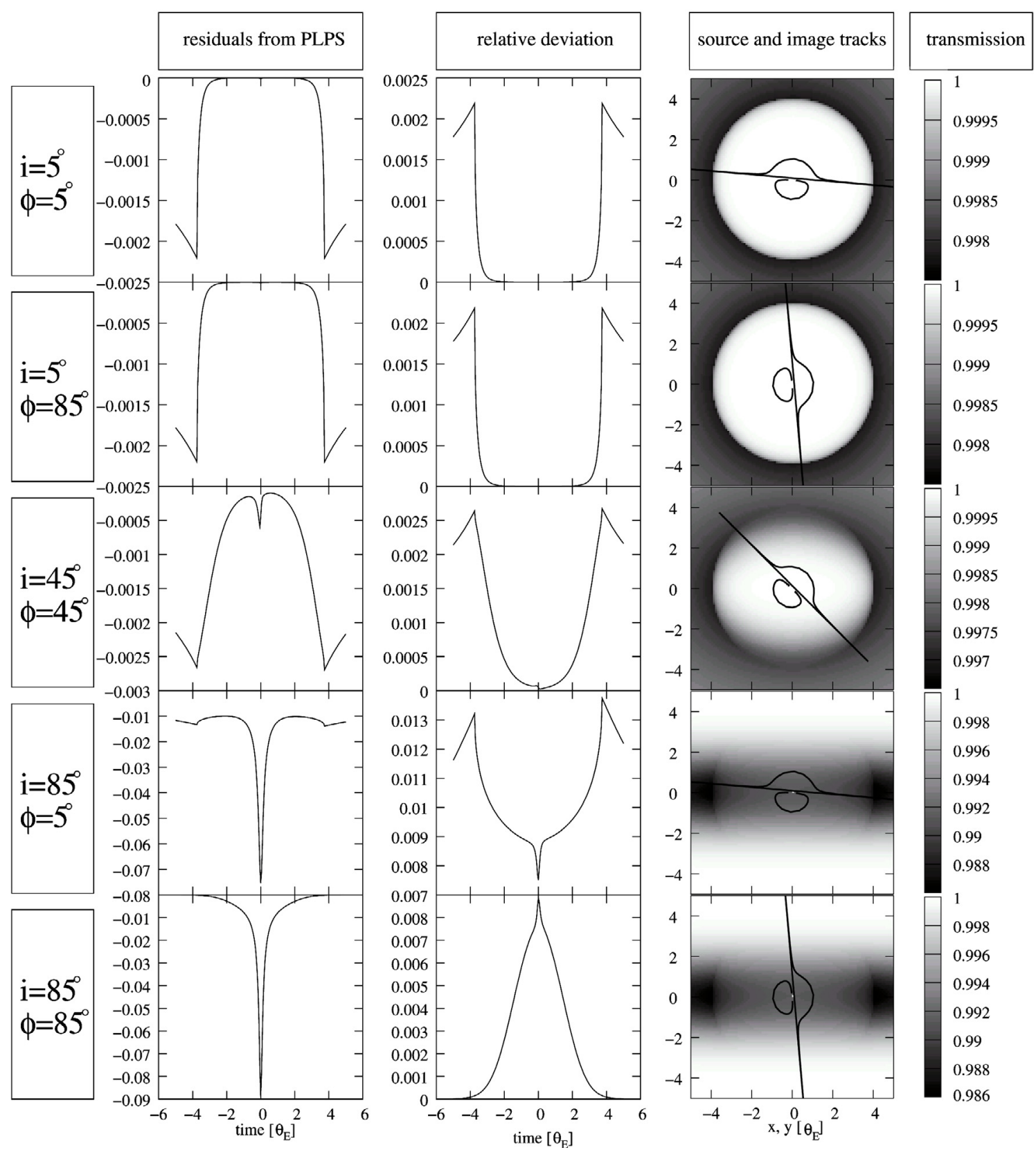

Fig. 5. Differential light curves of a geometrically thick disk with a central hole are shown for different inclinations $i$ and orientations $\phi$ of the source track. The disk has an inner radius of $10 \mathrm{AU}$ and an outer radius of $50 \mathrm{AU}, \Sigma \propto r^{-0.8} ; H=2.5 \mathrm{AU}$, and the average optical depth $\tau \approx 5 \times 10^{-4}$

indicates one possibility of detecting circumstellar disks - fitting a PSPL model and analyzing the structure of the residuals within a few Einstein radii. The simulated light curves show deviations between $0.1 \%$ and $1 \%$ (i.e. 1 and 10 mmag without blending flux), a signal that is detectable by a dedicated space mission like the Microlensing Planet Finder or EUCLID.

\section{Relative extinction curves}

It is possible to tell a priori whether a certain microlens is surrounded by attenuating matter if a wavelength-dependent extinction signal can be seen. When fitting a PSPL model, the residuals can be used to show where relative attenuation in the lens plane occurs between images taken at different wavelengths. A combination of photometric and astrometric follow-up could then infer the extinction structure of the central zone by measuring the extinction coefficient for each image: the centroid of the lensed source star will move slightly to the less dimmed image. The centroid $\boldsymbol{\theta}_{\mathrm{c}}$ with extinction is located at

$\boldsymbol{\theta}_{\mathrm{c}}=\frac{k_{+} \boldsymbol{\theta}_{+} \mu_{+}+k_{-} \boldsymbol{\theta}_{-} \mu_{-}}{k_{+} \mu_{+}+k_{-} \mu_{-}}$

where $k_{ \pm}$denote the measured transmission coefficients, $\mu_{ \pm}$the unextincted magnifications, $\boldsymbol{\theta} \pm$ the image positions and $\boldsymbol{\theta}_{\mathrm{c}}$ the trajectories on the sky. The total magnification is described as

$\mu=k_{+} \mu_{+}+k_{-} \mu_{-}$.

One of the transmission coefficients can be calculated from Eqs. (12) and (13):

$k_{-}=\frac{\mu}{\mu_{-}} \frac{\theta_{\mathrm{c}}-\boldsymbol{\theta}_{+}}{\boldsymbol{\theta}_{-}-\boldsymbol{\theta}_{+}}$ 
Ideally, brightness and position are measured simultaneously and thus the measured magnification $\mu$ and position $\boldsymbol{\theta}_{\mathbf{c}}$ can be jointly used in Eq. (14), after correcting for blended flux and baseline. Finally $k_{+}$is calculated from Eq. (13) by using the image positions $\boldsymbol{\theta}_{ \pm}$from the fitted PSPL model.

\section{Conclusions}

We have shown that gravitational microlensing can - in principle - be used as a tool for detecting circumstellar disks beyond $1 \mathrm{kpc}$ when the photometric residuals relative to a PSPL model are searched for deviations between -3 and $3 \theta_{\mathrm{E}}$ around the maximal magnification. We estimate that $4 \%$ of all $\mathrm{F}, \mathrm{G}$, \& K-microlenses contain debris disks. However, if the optical depth $\tau \geq 5 \times 10^{-4}$ (age $\sim 1$ Gyr), a tenth of these objects should have detectable disks. Assuming a power-law model for the surface density $\Sigma \propto r^{-0.8}$ and depending on the inner and outer radii distribution of debris disks, between 10 and $50 \%$ of all remaining light curves could show deviations greater than $1 \%$. For $\sim 4000$ existing light curves from the OGLE collaboration, we expect that around 10 of the F, G \& K-lenses show a detectable signature induced by a debris disk. If we consider that anomalous microlensing events, i.e. events that are not well-described by a PSPL model, can also display systematic variations and blending occurs, it is unlikely that debris disks can be discovered from the existing datasets. Nevertheless, future space missions will almost certainly provide the required accuracy for detecting debris disks and probing the attenuating matter.

Acknowledgements. This work was inspired by the Wilhelm und Else Heraeus Physics School "The Early Phase of Planet Formation". M.H. would like to acknowledge the support by the German-Israeli Foundation and from the Graduiertenkolleg 1351 .

\section{References}

Abe, F., Allen, W., Banks, T., et al. 1997, Variables Stars and the Astrophysical Returns of the Microlensing Surveys, 75

Abramowitz, M., \& Stegun, I. A. 1972, Handbook of Mathematical Functions (New York: Dover), 1972
Augereau, J.-C., \& Beust, H. 2006, A\&A, 455, 987

Aumann, H. H., Beichman, C. A., Gillett, F. C., et al. 1984, ApJ, 278, L23

Beaulieu, J.-P., Bennett, D. P., Fouqué, P., et al. 2006, Nature, 439, 437

Beaulieu, J. P., Kerins, E., Mao, S., et al. 2008 [arXiv : 0808.0005]

Bennett, D. P., Bond, I., Cheng, E., et al. 2004, Proc. SPIE, 5487, 1453

Bennett, D. P., Bond, I. A., Udalski, A., et al. 2008, ApJ, 684, 663

van den Bergh, S. 1990, JRASC, 84, 410

Boden, A. F., Shao, M., \& van Buren, D. 1998, ApJ, 502, 538

Bozza, V., \& Mancini, L. 2002, A\&A, 394, L47

Bozza, V., Jetzer, P., Mancini, L., \& Scarpetta, G. 2002, A\&A, 382, 6

Brauer, F., Dullemond, C. P., \& Henning, T. 2008, A\&A, 480, 859

Diehl, R., Halloin, H., Kretschmer, K., et al. 2006, Nature, 439, 45

Dominik, C., \& Decin, G. 2003, ApJ, 598, 626

Dominik, M. 2006, MNRAS, 367, 669

Gönner, H. 1996, Einführung in die spezielle und allgemeine Relativitätstheorie (Berlin: Spektrum Akademischer Verlag, Heidelberg)

Gould, A. 1992, ApJ, 392, 442

Greaves, J. S. 2005, Science, 307, 68

Han, C., \& Lee, C. 2002, MNRAS, 329, 163

Kalas, P., Liu, M. C., \& Matthews, B. C. 2004, Science, 303, 1990

Kayser, R., Refsdal, S., \& Stabell, R. 1986, A\&A, 166, 36

Krivov, A. V. 2005, LPI Contributions, 1280, 98

Krist, J. E., Ardila, D. R., Golimowski, D. A., et al. 2005, AJ, 129, 1008

Lindegren, L., \& Perryman, M. A. C. 1996, A\&AS, 116, 579

McCaughrean, M. J., \& O'dell, C. R. 1996, AJ, 111, 1977

McKee, C. F., \& Williams, J. P. 1997, ApJ, 476, 144

Metchev, S. A., Eisner, J. A., Hillenbrand, L. A., \& Wolf, S. 2005, ApJ, 622, 451

Paczynski, B. 1991, ApJ, 371, L63

Paczynski, B. 1998, ApJ, 494, L23

Rattenbury, N. J., \& Mao, S. 2006, MNRAS, 365, 792

Rhee, J. H., Song, I., Zuckerman, B., \& McElwain, M. 2007, ApJ, 660, 1556

Schneider, P., Ehlers, J., \& Falco, E. E. 1992, Gravitational Lenses, XIV (Berlin, Heidelberg, New York: Springer-Verlag)

Trilling, D. E., Bryden, G., Beichman, C. A., et al. 2008, ApJ, 674, 1086

Udalski, A., Szymanski, M., Kaluzny, J., et al. 1994, Acta Astron., 44, 227

Unwin, S. C., Shao, M., Tanner, Angelle, M., et al. 2008, PASP, 120, 38

Wambsganss, J. 1997, MNRAS, 284, 172

Wambsganss, J. 1999, J. Comput. Appl. Math., 109, 353

Wambsganss, J. 2006, Saas-Fee Advanced Course 33: Gravitational Lensing: Strong, Weak and Micro, 453

Wyatt, M. C., Smith, R., Greaves, J. S., Beichman, C. A., Bryden, G., \& Lisse, C. M. 2007, ApJ, 658, 569

Zheng, Z., \& Ménard, B. 2005, ApJ, 635, 599 